\title{
Ptosis and Mass Like Lesions in Behçet's Disease: A Rare Presentation
}

\author{
Seher KIR, ${ }^{1}$ Lütfi AKYOL, ${ }^{2}$ Metin ÖZGEN, ${ }^{2}$ Hediye PINAR GÜNBEY, ${ }^{3}$ Mehmet SAYARLIOĞLU ${ }^{2}$ \\ ${ }^{1}$ Department of Internal Medicine, Ondokuz Mayıs University, Faculty of Medicine, Samsun, Turkey \\ ${ }^{2}$ Department of Rheumatology, Ondokuz Mayıs University, Faculty of Medicine, Samsun, Turkey \\ ${ }^{3}$ Department of Radiology, Ondokuz Mayıs University, Faculty of Medicine, Samsun, Turkey
}

\begin{abstract}
In this article, we describe a case of neuro-Behçet's disease presenting with unilateral ptosis and facial paresis due to an intracranial mass lesion. A 25-year-old male patient with a history of Behçet's disease presented with headache, vertigo, double vision, ptosis in his right eyelid and slurred speech. Cranial magnetic resonance imaging scan revealed a right-sided capsulothalamic lesion, which was extending to the right cerebral pedicle, pons and superior cerebellar pedicle. This lesion was interpreted as neuro-Behçet's disease involvement of central nervous system. Cerebral masslike lesion is a rare form of neuro-Behçet's disease. Review of the literature revealed a limited number of cases. Ptosis related with Behçet's disease is also a very rare presentation. This case shows that this symptom might be a part of the clinical presentation of neuro-Behçet's disease.

Keywords: Behçet's disease; neuro-Behçet; ptosis.
\end{abstract}

Behçet's disease (BD) is a chronic, relapsing, inflammatory disorder, characterized by recurrent oral ulcers, skin lesions, genital ulcers, and uveitis. Its etiology is unknown and the major pathology is the presence of vasculitis. $\mathrm{BD}$ is a multisystem disorder because of the involvement of many organs. Although central nervous system (CNS) involvement is rare, it is a serious complication causing severe morbidity and mortality. ${ }^{1,2}$ Cerebral mass-like lesion is a rare form of neuro-Behçet's disease (NBD). A limited number of cases of NBD presenting with intracranial mass like lesions and also ptosis were reported in the literature. In this article, we report a patient with NBD presenting with unilateral ptosis due to an intracranial mass lesion. We believe that the publication of this case report may contribute to the literature in determining the true incidence of these cases.

\section{CASE REPORT}

A 25-year-old male patient was transferred to our hospital from emergency service of an another hospital with complaints of headache, vertigo, double vision, ptosis in his right eyelid and slurred speech that were present for the last three days. In addition, dysphagia and gait imbalance causing difficulty in walking were added for the last two days. His headache was continuous and severe. It started from forehead and radiated to whole head. There was no fever or projectile vomiting. Approximately one year before, he was diagnosed as BD because of his oral and genital ulcers by a dermatologist and $2 \times 0.5 \mathrm{mg} /$ day colchicine treatment was started. After that, the patient used colchicine irregularly and his oral ulcers repeated intermittently. Patient had no history of thrombophlebitis, deep vein thrombosis or seizures 
previously. His lumbar puncture was performed in the former hospital and a mild lymphocytic pleocytosis combined with an elevated protein level was detected (cerebrospinal fluid protein: $169 \mathrm{mg} / \mathrm{dL}$ [normal range: 15-45 mg/dL]). A written informed consent was obtained from the patient.

On clinical examination, he was conscious (Glasgow Coma Score: 15), his cooperation and orientation were full. There was ptosis in his right eye (Figure 1) and neurologic examination revealed third cranial nerve palsy on the right side, central facial paralysis and hemihypoesthesia on the left side of his face. Also, there was dysmetria and dysdiadochokinesia on the left side of his body. His right pupil was dilated and unreactive while there was no bilateral papilledema. Muscle strength was intact $(5 / 5)$, deep tendon reflexes were normoactive and plantar reflexes gave flexor response bilaterally. He had a history of uveitis attack two months before but his eye examination was normal for this time. Other systemic physical examinations were normal.

Sedimentation rate was $22 \mathrm{~mm} /$ hour (range 0-20 $\mathrm{mm} /$ hour) and C-reactive protein was $55 \mathrm{mg} / \mathrm{L}$ (0-3.5 mg/L). Routine laboratory tests such as complete blood count, peripheral blood smear, thyroid, renal and hepatic function tests and blood electrolytes were all normal. Tuberculosis polymerase chain reaction, antihuman immunodeficiency virus, hepatitis markers, TORCH panel, anti-nuclear antibody (ANA), and ANA subgroups were negative.

A contrast-enhanced cranial magnetic resonance imaging (MRI) was performed, which demonstrated a right-sided capsulothalamic lesion which was mild hypointense in $\mathrm{T}_{1}$-weighted images and hyperintense in $\mathrm{T}_{2}$-weighted/fluid attenuated inversion recovery images and extending from thalamus to the right cerebral pedicle, pons and superior cerebellar pedicle (Figure 2a-c). Peripheral patchy contrast enhancement was

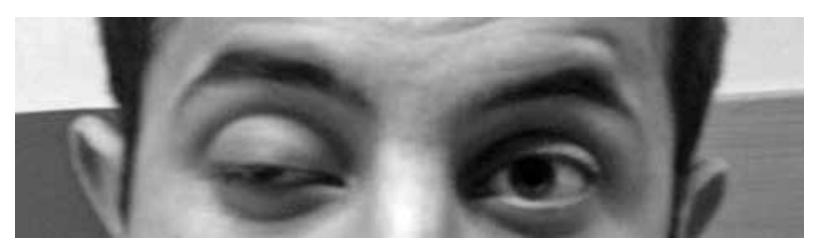

Figure 1. Unilateral ptosis in right eye. seen in thalamus and right cerebral pedicle in mesencephalon following contrast injection (Figure 2d). This lesion was interpreted as $\mathrm{NB}$ involvement of CNS. In diffusion MRI, defined lesion showed iso-hyperintense areas in diffusion-weighted images and low apparent diffusion coefficient. There were millimetric contrast-free parietal subcortical white matter lesions bilaterally in centrum semiovale and corona radiata level. Diffusion MRI findings were also interpreted as vasculitic lesions. Contrastenhanced computed tomography (CT) and $\mathrm{CT}$ angiography of abdomen and thorax were performed for the exclusion of any thrombus or aneurysm and no abnormalities were observed.

Patient was treated with intravenous $1 \mathrm{~g}$ pulse steroid for seven days and continuous subcutaneous low weight heparin injections. Because of the presence of poor prognostic factors, cyclophosphamide treatment was planned. In the follow-up, clinical improvement was achieved and patient was discharged from hospital. Control cranial MRI could not be performed due to losing contact with the patient.
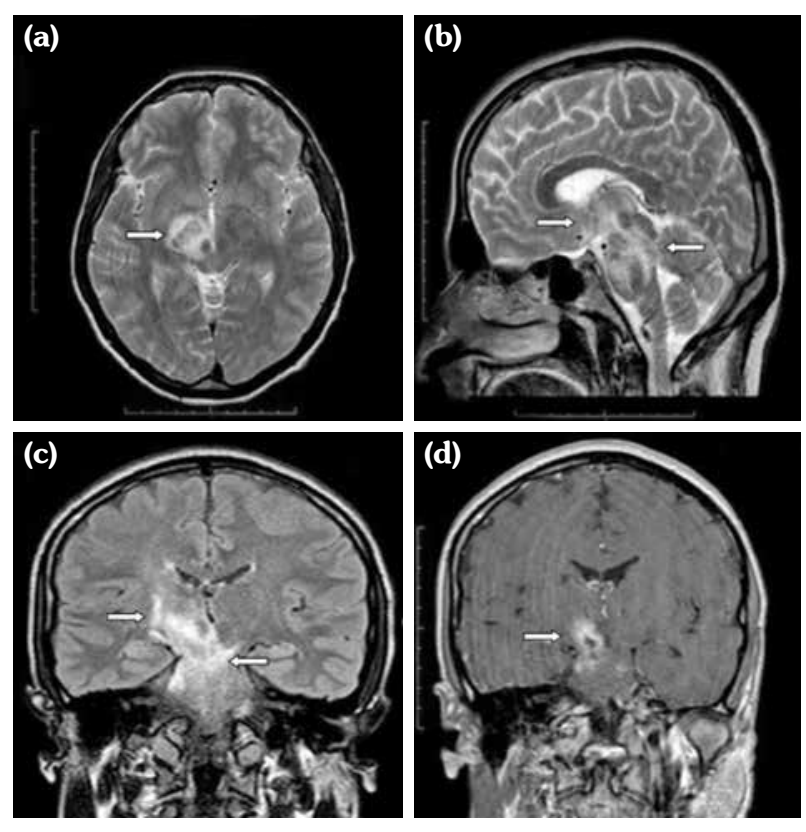

Figure 2. Magnetic resonance imaging scan: (a) $\mathrm{T}_{2}$-weighted axial, sagittal and (b) fluid attenuated inversion recovery coronal (c) images show a right-sided hyperintense capsulothalamic lesion extending to right cerebral pedicle, pons and superior cerebellar pedicle. (d) Peripheral patchy contrast enhancement is seen in thalamus and right cerebral pedicle in mesencephalon following contrast injection. 


\section{DISCUSSION}

Involvement of CNS is reported to occur in $2.2-50 \%$ of patients with $\mathrm{BD}$ and it is more common in males with early onset BD. ${ }^{2,3}$ CNS involvement includes parenchymal and nonparenchymal lesions. Clinical features frequently consist of findings of cranial nerve palsies, cerebellar ataxia, and corticospinal tract involvement. Parenchymal involvement is seen more (82\%) and lesions are located mostly in brainstem, diencephalon, basal ganglion, and internal capsule.

Parenchymal involvement in the form of mass-like lesion is a rare presentation and a limited number of cases were reported in the literature..$^{2,4-6}$ In contrast to classical NBD, patients with mass-like lesions mainly have one intracerebral space-occupying large lesion which is often found in the capsulothalamic area and "typical" radiological findings are infrequently reported. ${ }^{7,8}$ Noel et al. ${ }^{6}$ reported the first case series of mass-like form of NBD, including five personal observations and a review of 18 cases from the literature. They estimated the prevalence of the mass-like form of NBD as 1.8\% in their study group (275 patients with NBD). ${ }^{6}$ Later, Bilge et al. ${ }^{2}$ and Tokgoz et al. ${ }^{5}$ added two new cases to the literature.

Non-parenchymal involvement (neurovascular BD) includes dural sinus thrombosis, arterial thrombosis, and aneurysms. Both involvements may be seen rarely in the same person. ${ }^{8}$

Ptosis and fascicular oculomotor nerve involvement are rarely observed in BD..$^{1,9,10}$ Third nerve palsies may be partial or complete and may result from lesions located anywhere from the oculomotor nucleus to the termination of the third nerve in the extra-ocular muscles within the orbit. Symptoms can be related isolatedly to the third nerve palsy or accompanied by signs of other neurological findings. ${ }^{11}$ Most of the third nerve palsies are of ischemic etiology. Presence of unilateral ptosis in our patient was suggesting third cranial nerve involvement, which is a very rare presentation of NBD. ${ }^{1,9,10}$ Reported cases defined fascicular oculomotor nerve palsy secondary to $\mathrm{NBD}$, and the lesions were, as in our case, extending from mesencephalon to the capsulothalamic area. Upgaze and oculomotor nerve palsy were suggested to be caused by the mass effect of the lesion and fascicular involvement. ${ }^{9,10}$

Histopathological examination is recommended to rule out infectious lesions (bacterial abscess or tuberculoma), malignant etiologies (cerebral glioblastoma or lymphoma), and inflammatory conditions (sarcoidosis, multiple sclerosis, Wegener's disease) and to avoid inadequate use of immunosuppressive agents. ${ }^{8}$ However, the risk of neurological sequelae is increased with delayed diagnosis; therefore, we did not consider brain biopsy for diagnosis due to prolonged pathological examination time.

Cerebrospinal fluid examination is needed in differential diagnosis of parenchymal NBD and multiple sclerosis. In general, slightly moderate lymphocyte and polymorphonuclear cell proliferation along with moderate concentrations of protein levels are observed in NBD, as in our case. ${ }^{5}$ So, we started immediate treatment in the light of prior BD diagnosis of our patient.

In acute attack, a high dose corticosteroid ( $1 \mathrm{~g} /$ day) is mostly used for treatment of NBD. There is no accepted specific treatment for the maintenance period but some authors suggested a life-long colchicine (1-2 mg/day), acetylsalicylic acid, and low dose corticosteroid treatments. ${ }^{2,3,12}$ In published cases, the most common maintenance therapy was immunosuppression with cyclophosphamide or azathioprine with a dramatic improvement in clinical and radiologic manifestations in $83 \%$ of patients. ${ }^{2,3,12} \mathrm{We}$ also planned cyclophosphamide treatment after the intravenous pulse steroid.

In conclusion, neurologic presentations of $\mathrm{BD}$ are various and poorly understood. Neurologic involvement in $\mathrm{BD}$ may be either prior to or after the $\mathrm{BD}$ diagnosis. We need to consider NBD in patients with neurologic findings particularly with supporting MRI lesions. Therefore, early detection of NBD and beginning urgent treatment may prevent any neurologic sequela and increase life quality of patients.

\section{Declaration of conflicting interests}

The authors declared no conflicts of interest with respect to the authorship and/or publication of this article. 


\section{Funding}

The authors received no financial support for the research and/or authorship of this article.

\section{REFERENCES}

1. Matsui $T$, Ishida $T$, Tono $T$, Yoshida $T$, Sato $S$, Hirohata S. An attack of acute neuro-Behçet's disease during the course of chronic progressive neuroBehçet's disease: report of two cases. Mod Rheumatol 2010;20:621-6.

2. Bilge NŞ, Şaylısoy S, Kaşifoglu T, Korkmaz C. Masslike lesions as a rare form of neuro-Behçet's disease: A case report and review of the literature. Eur $\mathrm{J}$ Rheumatol 2014;1:34-8.

3. Diri E, Espinoza LR. Neuro-Behçet's syndrome: differential diagnosis and management. Curr Rheumatol Rep 2006;8:317-22.

4. Yoshimura S, Ago T, Koga M, Kamouchi M, Kitazono T. Cerebral Small-Vessel Disease in Neuro-Behçet Disease. J Stroke Cerebrovasc Dis 2015;24:e237-9.

5. Tokgoz OS, Akpinar Z, Guney F, Seyithanoğlu A. A Neuro-Behçet's Case Operated with the Intracranial Mass Misdiagnosis. J Korean Neurosurg Soc
2012;52:488-90

6. Noel N, Hutie M, Wechsler B, Vignes S, Le Thi Huong-Boutin D, Amoura Z, et al. Pseudotumoural presentation of neuro-Behcet's disease: case series and review of literature. Rheumatology (Oxford) 2012;51:1216-25.

7. Kalra S, Silman A, Akman-Demir G, Bohlega S, Borhani-Haghighi A, Constantinescu CS, et al. Diagnosis and management of Neuro-Behçet's disease: international consensus recommendations. $\mathrm{J}$ Neurol 2014;261:1662-76.

8. Borhani Haghighi A, Pourmand R, Nikseresht AR. Neuro-Behçet disease. A review. Neurologist 2005; 11:80-9.

9. Saribas O, Aydin-Kirkali P, Erdem E, Calgüner M. Fascicular oculomotor nerve palsy in neuro-Behçet's disease. J Clin Neuroophthalmol 1991;11:300-5.

10. Masai H, Kashii S, Kimura H, Fukuyama $H$. Neuro-Behçet disease presenting with internuclear ophthalmoplegia. Am J Ophthalmol 1996;122:897-8.

11. Biousse V, Newman NJ. Third nerve palsies. Semin Neurol 2000;20:55-74.

12. Imoto $H$, Nishizaki $T$, Nogami $K$, Sakamoto $K$, Nomura S, Akimura T, et al. Neuro-Behçet's disease manifesting as a neoplasm-like lesion--case report. Neurol Med Chir (Tokyo) 2002;42:406-9. 